\title{
Liberté reproductive et recours à la contraception : les influences religieuse et sociale au Niger
}

\author{
Abdoul Moumouni Nouhou \\ Institut de Démographie et de Socioéconomie, Université de Genève
}

nouhouabdoulm@gmail.com

\section{Résumé}

Cette étude examine le rôle de l'entourage social et de la religion sur l'opinion des femmes nigériennes au sujet du contrôle de naissances. Elle utilise des données collectées par une enquête réalisée en 2014 auprès d'un échantillon de 2004 femmes âgées de 15 à 49 ans. Les résultats montrent que les femmes approuvent le contrôle des naissances lorsqu'il s'entend comme un recours à la contraception moderne pour espacer les naissances, mais non comme un choix du nombre d'enfants à avoir, au prix d'une éventuelle limitation des naissances. L'entourage social et la religion jouent un rôle important sur l'acceptation du contrôle de naissances. Les consoeurs de la communauté influencent principalement les femmes moins religieuses alors que les plus religieuses sont surtout influencées par leur mari et les leaders religieux. Cependant, la religiosité de la femme ne détermine pas son expérience contraceptive. Celle-ci dépend surtout de ses attitudes face au contrôle de naissances.

Mots clés: entourage social, religiosité, femmes, contrôle des naissances, Niger

\section{Abstract}

\section{Reproductive freedom and contraception use: religious and social influences in Niger}

This paper studies the role of the social context and of religiosity on the opinions of Niger women about birth control. We use data from a survey realized in 2014 on a sample of 2004 women aged I549 years. Results highlight that women do approve birth control when it means using modern contraception for birth spacing, but not for choosing their number of children and therefore the possible recourse to contraception for limiting births. Women's social context and religiosity significantly influence their acceptance of birth control. Less religious women are mainly influenced by other women from the community, whereas husbands and religious leaders are the main references of the more religious ones. Yet, women' religiosity does not determine their contraceptive experience which rather depends on their attitude towards birth control.

Keywords: social context, religiosity, women, birth control, Niger

\section{Introduction}

L'acception morale de l'idée d'une intervention délibérée visant à contrôler les naissances est l'une des trois pré-conditions d'un déclin durable de la fécondité énoncées par Coale (1973: 65). Connues par la suite sous le nom du modèle RWA (Ready, Willing and Able), ces trois conditions n'ont pas fait l'objet de la même attention dans l'explication de la transition féconde des pays en développement. La question de l'acceptation morale ou sociale du contrôle de naissances a très tôt été évacuée, sans doute parce qu'elle a aussi été peu présente dans les pays d'Amérique Latine et d'Asie, les premières régions en développement à entrer rapidement en transition après les années 1950. Les débats sur les avantages socioéconomiques d'une descendance plus ou moins nombreuse (deuxième condition) et l'importance de la disponibilité/accessibilité des moyens de contrôle des naissances (troisième condition) ont pris le pas sur l'étude du préalable au recours à la contraception, c'est-à-dire l'acceptation de l'idée même de contrôle des naissances comme un mode de pensée ou une forme de comportement.

Pourtant, la transition de la fécondité en Afrique est assez singulière comparativement aux autres régions du monde, notamment par sa lenteur et les raisons qui expliquent sa rigidité. Dans un pays où la fécondité reste encore élevée et la prévalence contraceptive très faible comme le Niger, l'acception 
de l'idée de contrôle des naissances n'est pas une évidence. D'après l'Enquête démographique et de santé (INS et Macro International Inc., 2007), parmi les femmes nigériennes de 15 à 49 ans non utilisatrices de la contraception, 29\% n'ont pas l'intention de l'utiliser à l'avenir parce que soit elles, soit leurs maris ou d'autres membres de leur famille s'y opposent et $6 \%$ ne comptent pas l'utiliser pour des raisons religieuses. Une faible part d'entre elles déclarent n'avoir pas l'intention de l'utiliser à l'avenir pour des raisons sanitaires (3\%) ou par crainte des effets secondaires (2\%). II subsiste donc une opposition "de principe" à la contraception comme le montre ces références à la religion et à l'opinion de l'entourage social comme motifs de rejet. Cette opposition, dite de principe, mérite une attention particulière dans les pays à forte fécondité en particulier les pays musulmans.

En s'inspirant de la théorie du comportement planifié (Ajzen, 1991), cette étude examine d'abord la place de l'entourage social et de la religion dans l'opinion des femmes sur le contrôle de naissances. Elle analyse ensuite si et comment l'acceptation (ou le rejet) du contrôle de naissances et le degré de religiosité sont liés à l'expérience contraceptive de la femme.

\section{Revue de la littérature et Cadre théorique}

\section{Principaux courants}

La littérature sur les liens entre la religion et les comportements reproductifs se résume en trois principales théories ou hypothèses: l'hypothèse dite des caractéristiques, l'hypothèse de la théologie particularisée et l'hypothèse d'interactions. L'hypothèse des caractéristiques (Goldscheider, 1971) stipule que les différentiels de fécondité entre groupes religieux s'expliquent exclusivement par les différences de caractéristiques socioéconomiques et démographiques entre ces groupes. II n'existerait donc pas d'effet religieux intrinsèque sur les comportements reproductifs. A l'opposé, l'hypothèse de la théologie particularisée (Goldscheider, 197I) postule que la variation des comportements reproductifs entre différents groupes s'explique plutôt par un effet différentiel de leurs doctrines religieuses. L'idée centrale est ici que, selon la nature des valeurs qu'elles promeuvent, certaines religions sont plus pronatalistes que d'autres. Les études qui expliquent le niveau de fécondité d'un groupe à travers la religion de ses adeptes adoptent implicitement cette posture (Frejka et Westoff, 2008 : 28). Chamie (198I) postule que les comportements reproductifs sont déterminés par une synergie entre la religion et les conditions socioéconomiques: c'est l'hypothèse dite d'interactions, laquelle constitue un compromis entre les deux hypothèses de Goldscheider (197I).

A ce trio peut s'annexer l'hypothèse de statut de groupe minoritaire (Goldscheider, 197I). Celle-ci répond à la nécessité d'une prise en compte de "l'organisation sociale des communautés religieuses en tant qu'objet d'analyse, notamment s'il s'agit des groupes minoritaires" (Ambrosetti, 20II). Sous certaines conditions énumérées par Moulasha et Rao (1999 : 3047), l'insécurité à laquelle est soumis un groupe minoritaire pourrait réduire sa fécondité en dessous de celle du groupe majoritaire. D'une façon générale, cette hypothèse suppose des comportements reproductifs propres pour une classe sociale minoritaire et religieusement définie.

Au-delà des postulats sur les liens entre la religion et la fécondité, Simons (1982 : 383) se propose d'analyser la fécondité comme une pratique religieuse. Plus qu'une conséquence, les comportements reproductifs relèvent directement de la religion comme dans l'exemple des Huttérites. Le balisage de la vie reproductive en Islam (procréation dans le cadre strict du mariage; contrôle des naissances sous conditions, voire interdit; l'enfant comme une bénédiction, un cadeau de Dieu; etc.) rappelle cette vision.

Le modèle de religiosité islamique de Krauss et al (2006 : 240) (The Muslim Religiosity-Personality Inventory) le montre, au-delà du rapport de l'homme à Dieu (Ibadah), I'Islam dicte également le rapport de l'homme à son prochain (Mu'amalat) et donc à son entourage. Les rapports de la femme à son mari, à sa famille et plus largement à sa communauté peuvent affecter ses opinions et comportements à propos du contrôle des naissances. Dans un pays où cohabitent les valeurs familiales traditionnelles et les valeurs islamiques, les rôles de l'entourage social et de la religion sur les comportements reproductifs s'entremêlent (Sounaye, 20II). Cependant, malgré une structure patriarcale de la famille et un environnement peu propice à l'autonomisation, l'opinion des femmes -et dans une certaine mesure leurs comportements- évoluent et se diversifient (Moussa, 2004 : 3I), suivant notamment leurs caractéristiques socioéconomiques et démographiques propres.

\section{Opinions et comportements sous pression}

L'acception (ou le refus) du contrôle de naissances peut résulter des convictions religieuses ou de la pression sociale exercée sur la femme. D'un point de vue religieux, la position de la femme oscille entre deux perceptions de la procréation: une vision "fondamentaliste" et une vision "pragmatique" (Simons, 1982 : 391-392). Caractéristique d'un individu plus religieux, la première est sacrée. La 
seconde est une vision profane, spécifique à un individu plus indépendant de la religion dans ses choix en matière de reproduction.

La position de la femme est aussi déterminée en partie par son entourage social. Malgré l'émancipation féminine en cours dans les pays du sud (Adjamagbo et Calvès, 2012 : 12), le poids d'une structure familiale patriarcale et de certaines normes sociales continuent de peser sur la liberté des femmes d'effectuer des choix libres en matière de procréation. La volonté de satisfaire les attentes de son entourage peut parfois la conduire à renoncer à certaines options, à certains droits. A ce propos, le modèle d'influence sociale (Montgomery et Casterline, 1996 : 155-156) postule que les décisions individuelles en matière de fécondité sont affectées par des contraintes non pas simplement monétaires ou matérielles, mais aussi d'ordre social. Les groupes sociaux (référents) que la femme côtoie quotidiennement définissent un vecteur $d^{\prime}$ 'information ${ }^{\text {ji }}$ qui lui sert pour les choix de comportements à adopter. Ainsi, les comportements reproductifs dépendent partiellement du "degré auquel les femmes en âge de procréer sont capables d'agir en leur nom propre" (Mason, 1987 : 447). Finalement, l'adoption d'un comportement innovant (recours à la contraception par exemple) dépend de la capacité de la femme à contenir les pressions sociales et religieuses, ce qui dépend en partie de ses propres caractéristiques socioéconomiques et démographiques (niveau d'instruction, statut économique, autonomie, etc.).

\section{Vers la théorie du comportement planifié}

Les barrières d'accès à la planification familiale dans les pays en développement sont bien identifiées (Campbell et al, 2006 : 87-93). Par contre, peut-être parce qu'elles s'apprêtent mieux à faire l'objet d'intervention, les contraintes liées à l'offre semblent plus étudiées que celles liées à la demande. Ces dernières sont en général de nature socioculturelle et relèvent des systèmes de valeurs (tradition, religion, etc.). Or, Stephenson et Hennink (2004 : 15) montrent que quand la femme reste sous le contrôle de son mari ou de sa belle famille, les barrières psychosociales peuvent s'avérer plus cruciales que les problèmes d'accessibilité géographique ou économique à la contraception. Comme le soulignent Casterline et al (200I : 98), les coûts socioculturels du recours à la contraception, y compris l'attitude du mari, réduisent considérablement la demande et l'utilisation des services de planification familiale. L'entourage social constitue ainsi une entrave à l'adoption de ce que Sauvain-Dugerdil et al (2014 : 857) appelle la "culture contraceptive". Même quand la femme a un accès garanti aux moyens contraceptifs, leur utilisation dans la durée dépend en partie du consentement de son entourage familial, son mari au premier rang (Cotten et al, 1992 : 147).

Le rôle des personnes externes au cercle familial ne serait pas non plus négligeable. Au cours d'une discussion de groupe iii, quand une participante mentionne son intention d'espacer ses naissances pour parachever ses études et devenir enseignante, la réaction d'une autre participante est inattendue: "Dans les cinq années à venir dont tu parles, nous voulons te voir avec 4 enfants, en plus de ton premier enfant qui est là. Cela te fera cinq (5) enfants, un beau monde autour de toi et tu verras que c'est encore meilleur qu'une maitresse d'école -rires-". Dans le cadre de la même enquête, la référence aux leaders religieux pour justifier un comportement (refus de la contraception, obéissance au mari, etc.) est récurrente.

Du mari aux autres femmes de la communauté, en passant par la famille et les leaders religieux (référents sociaux), les femmes composent avec les attentes qu'elles perçoivent de tous. L'ensemble de ces personnes autour d'elles constituent sa référence sociale pour le contrôle des naissances et définissent ses croyances normativesiv. Dans le cadre de la théorie du comportement planifié (TCP) (Ajzen, 199I; Ajzen et Klobas, 2013 : 206), l'ensemble de ses croyances normatives forme ce que les auteurs appellent "la norme subjective". Cette dernière mesure la pression sociale, positive ou négative, qui détermine en partie les intentions de la femme par rapport à un comportement donné. 
Figure I - Adaptation du cadre de la théorie du comportement planifié (Ajzen, I99I; Ajzen et Klobas, 20I3:206; Chauvin et al, 2007)

\begin{tabular}{|l|}
\hline Facteurs \\
socioéconomiques et \\
démographiques \\
Age, \\
Nombre d'enfants, \\
Niveau d'instruction, \\
Autonomie dans le \\
ménage, \\
Niveau d'instruction du \\
mari, \\
Statut économique. \\
Facteurs sociétaux \\
Religion (Religiosité) \\
Milieu de résidence
\end{tabular}

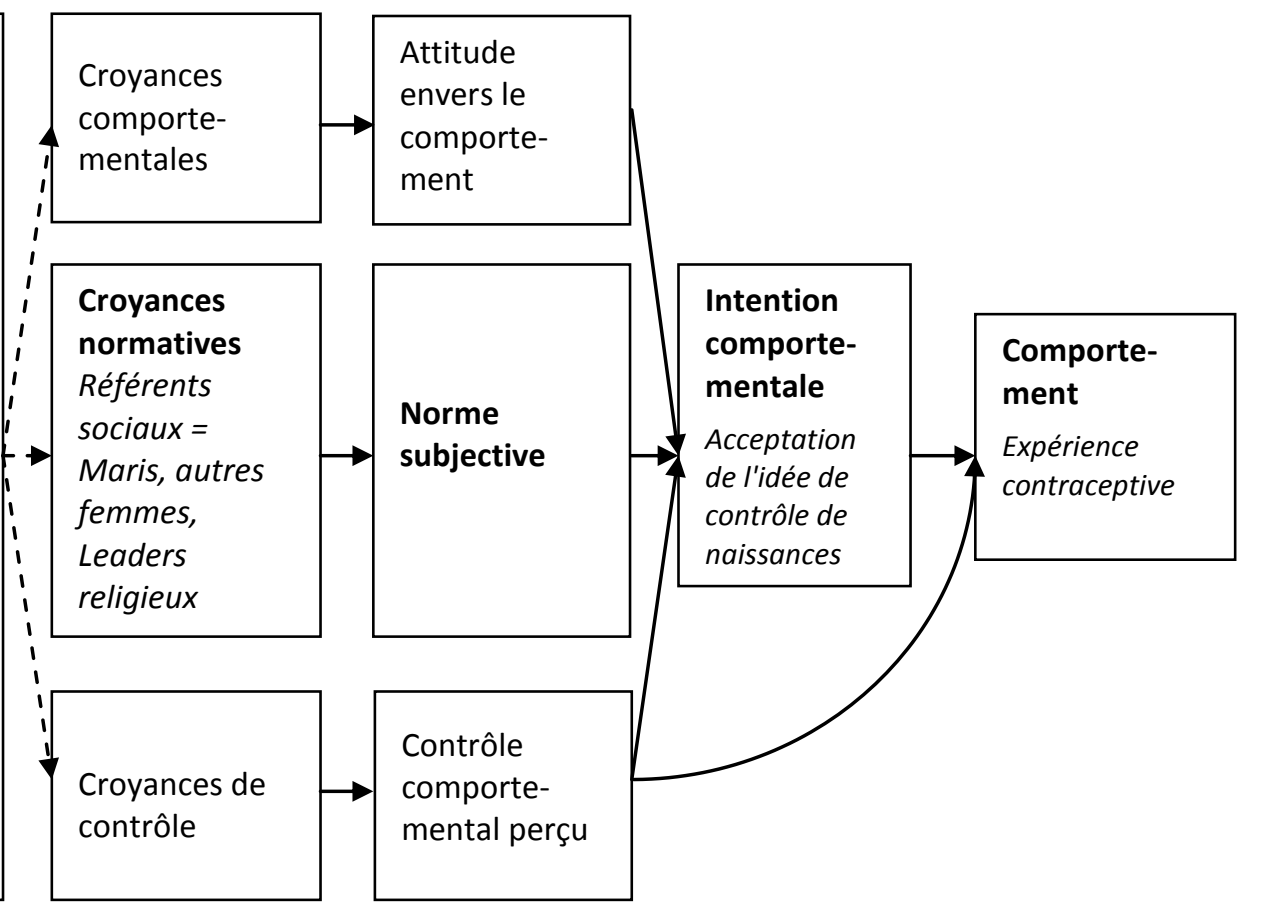

Avec l'expérience contraceptive comme comportement cible, nous adaptons le schéma de la TCP en appréhendant les intentions comportementales par les opinions de la femme sur le contrôle des naissances. Les liens entre les déterminants de l'intention, l'intention et le comportement qui en résulte n'ont de sens que dans un contexte précis, définit par les normes sociales et culturelles. Comme le cadre de la TCP est applicable en deux étapes (Chauvin et al, 2007 : 38), nous analysons d'abord les liens entre les opinions de la femme et ses croyances normatives et, ensuite, les rapports entre les opinions de la femme et son expérience contraceptive. Ces rapports entre "intentions" et comportement ne sont pas mécaniques comme le soulignent de nombreuses études longitudinales (Monnier, 1987 : 834; Toulemon et Testa, 2005 : 2-3; Régnier-Loilier et Vignoli, 20I I : 4l6).

\section{Hypothèses}

La perception des attentes de son entourage social définit pour la femme un espace de liberté d'opinions en matière de procréation. La religion conditionnerait cette perception, contribuant ainsi à la détermination de cet espace de liberté et donc à l'opinion de la femme à propos du contrôle de naissances.

On peut naturellement concevoir que les femmes qui approuvent le contrôle de naissances soient plus enclines à l'usage de la contraception. Mais quand le conjoint, la famille proche, voire l'ensemble de la société concourent à la sélection des comportements reproductifs à adopter, cette logique est moins évidente. Les Nigériennes auraient ainsi des difficultés à aligner leur expérience contraceptive réelle à leurs pinions à l'égard du contrôle de naissances. Leur expérience contraceptive ne dépendrait pas de leurs opinions, mais plutôt de leurs caractéristiques socioéconomiques et de leur degré de religiosité, dans une sorte de gestion d'incertitudes (Johnson-Hanks, 2005 : 366-377).

\section{Données et méthodes}

\section{Données et source}

Mettant un accent particulier sur la religiosité en Islam et les comportements reproductifs, une enquête quantitative récemment réalisée (en 2014) au Niger par Hope Consulting 'vonne l'opportunité de mener des analyses plus aiguës sur le rôle de la religion dans la sphère reproductive. Les données récoltées par cette enquête sont riches et détaillées, aussi bien sur les questions religieuses que sur les facteurs importants du recours à la contraception. Elles sont utilisées pour le besoin des analyses menées dans ce travail.

Calquée sur le modèle du plan d'échantillonnage de l'enquête EDS 2012, l'enquête susmentionnée a permis d'interroger un échantillon de 2004 femmes âgées de 15 à 49 ans dans six régions du pays ${ }^{\mathrm{vi}}$. Les analyses portent sur les femmes musulmanes (99\% de l'échantillon initiale). Pour un souci d'homogénéisation, les femmes célibataires (au nombre de 200) sont exclues de l'échantillon final (I78I femmes musulmanes mariées). En effet, le mariage conditionne systématiquement les comportements reproductifs des femmes et leur 
autonomie vis-à-vis de l'homme ne se définit que dans le cadre de cette union. De plus, le mariage compte parmi les signaux de la transition à l'âge adulte, avec des obligations religieuses plus strictes.

\section{Concepts et mesures}

\section{Religiosité}

Compte tenu des données disponibles, l'indicateur de religiosité proposé s'inspire de la définition de Glock $(1961)^{\text {vii. }}$. Trois des cinq dimensions proposées par Charles Glock sont reprises ici, à savoir les dimensions intellectuelle, ritualiste et conséquentielle. Sous l'hypothèse que les études coraniques favorisent une meilleure connaissance de l'Islam, la dimension intellectuelle est appréhendée par la fréquentation passée ou actuelle d'une école coranique. L'assiduité dans les prières quotidiennes renvoie à la dimension ritualiste et la dimension conséquentielle est saisie à travers la position de la femme par rapport à la diversité d'opinions en Islam et l'autorité des Imams ${ }^{\text {viii }}$.

Tableau I - Dimensions et variables de la religiosité

\begin{tabular}{|c|c|c|}
\hline Dimensions & Questions/variables & Modalités \\
\hline Intellectuelle & Avez-vous fait de l'éducation coranique? & $\begin{array}{l}\text { Oui (+) } \\
\text { Non (-) }\end{array}$ \\
\hline Ritualiste & Cela vous arrive-t-il de ne pas prier 5 fois par jour? & $\begin{array}{l}\text { Jamais }(+) \\
\text { Souvent }(+ \text { ou }-) \\
\text { Toujours }(-)\end{array}$ \\
\hline Conséquentielle & $\begin{array}{l}\text { Dans I'Islam, tout est clair; il n'y a pas de place pour une } \\
\text { diversité d'opinions } \\
\text { Les Imams et dirigeants religieux ont toute autorité sur } \\
\text { mon islam }\end{array}$ & $\begin{array}{l}\text { Oui }(+) \\
\text { Non }(-) \\
\text { Oui }(+) \\
\text { Non }(-)\end{array}$ \\
\hline
\end{tabular}

Après une Analyse factorielle des correspondances multiples (AFCM) sur les variables retenues, les trois premiers axes factoriels (68\% de l'inertie initiale) ont été retenus pour servir à une classification hiérarchique ascendante. A la lumière de l'Indice d'agrégation et de la Silhouette moyenne, trois niveaux de religiosité ont été retenus: les femmes moins religieuses $(53,3 \%)$, les plutôt religieuses $(21,3 \%)$ et les plus religieuses $(25,4 \%)$. Pour simplifier les analyses cependant, les catégories "moins" et "plutôt" religieuses sont fusionnées et traiter comme "moins religieuses".

\section{Statut économique de la femme}

Il est défini à partir des caractéristiques du ménage, notamment la possession d'articles électroménagers, y compris le réfrigérateur, l'ordinateur personnel et le téléphone fixe ou portable, etc. II intègre aussi le nombre de pièces dans la maison, les moyens de transport détenus par le ménage, l'exercice d'une activité rémunérée au cours des 12 derniers par la femme ainsi que le niveau d'instruction du chef du ménage au sein duquel habite la femme.

En procédant comme précédemment (AFCM et classification hiérarchique sur 6 des 17 axes factoriels, soit $53,43 \%$ de l'inertie initiale), deux groupes économiques ont été dégagés : un groupe majoritaire de femmes à faible statut (69\%) et un groupe moins nombreux de femmes plus nanties (31\%).
Contrairement aux autres, celles à statut économique plus élevé possèdent le plus souvent tous les équipements ou matériels électroménagers et leurs ménages vivent dans des maisons à plusieurs pièces, avec au moins une mobylette ou une voiture. Elles ont plus souvent exercé un emploi rémunéré au cours des 12 derniers mois et leurs ménages sont dirigés par un chef de niveau d'instruction plus élevé.

\section{Deux formes d'autonomie de la femme}

La participation de la femme à la prise des décisions au sein du ménage reste précaire au Niger. A titre illustratif, $3,5 \%$ des femmes seulement décident seules pour leurs soins de santé, 17,9\% conjointement avec leurs maris et pour $76,3 \%$ d'entre elles cette décision revient exclusivement à leurs maris. Pour les achats importants du ménage, ces chiffres sont respectivement de 3,2\% (femmes seules), $16,8 \%$ (décision conjointe) et $77,3 \%$ (maris seuls) (INS et Macro International Inc., 2007). Dans ce contexte, prendre conjointement avec le mari certaines décisions importantes pour le ménage est signe d'une certaine autonomie.

Dans ces conditions, est considérée comme plus autonome dans la gestion du ménage la femme qui cumule les trois avantages suivants: (a) prend seule ou avec son mari les décisions d'achats dans le ménage, (b) décide seule ou avec son mari des questions qui touchent à sa santé et (c) participe 
toujours ou souvent à la prise des décisions financières avec son mari. Ainsi, 35,4\% des femmes qui satisfont ces conditions sont considérées comme jouissant d'une certaine autonomie dans la gestion de leurs ménages.

Les femmes plus autonomes dans la gestion de leur sexualité sont celles qui prennent seules ou avec leurs maris les décisions relatives à la contraception dans le couple et qui peuvent, si elles le souhaitent, refuser un rapport sexuel avec leurs maris. Contrairement à l'autonomie dans la gestion du ménage, $10,3 \%$ seulement de femmes sont autonomes dans la gestion de leur sexualité.

\section{Référents sociaux, espace des libertés}

L'entourage social de la femme se compose de trois groupes sociaux de référence dont son mari, les autres femmes et les leaders religieux de sa communauté. La norme subjective est définie à partir de quatre croyances normatives qui correspondent aux quatre questions portant sur l'acceptation des pré-requis au contrôle des naissances (tableau 2). Les femmes sont d'abord invitées à donner leurs opinions sur ces questions (oui ou non), puis à préciser ce qu'elles pensent être les positions de leurs référents sociaux.

Les analyses descriptives mettent en parallèle les opinions de la femme aux perceptions qu'elle a des opinions de ses référents. L'influence de l'entourage est alors mesurée par la proximité (ou l'éloignement) entre ses opinions propres et les perceptions qu'elle a des opinions des autres. Pour la femme et chacun de ses référents, un indicateur synthétique ${ }^{\mathrm{ix}}$ appelé score de liberté cumule les opinions favorables sur les quatre questions posées, chaque opinion favorable comptant pour I point.

Tableau 2 - Référents sociaux et dimensions de la liberté reproductive

\begin{tabular}{|llllll|}
\hline & \multicolumn{4}{c|}{ Acceptation du contrôle des naissances } \\
\cline { 2 - 5 } $\begin{array}{l}\text { Femme et référents } \\
\text { sociaux }\end{array}$ & $\begin{array}{l}\text { I) Est-il acceptable } \\
\text { de choisir la taille de } \\
\text { sa descendance? }\end{array}$ & $\begin{array}{l}\text { 2) Est-il } \\
\text { acceptable } \\
\text { d'espacer les } \\
\text { naissances? }\end{array}$ & $\begin{array}{l}\text { 3) Est-il } \\
\text { acceptable de } \\
\text { limiter les } \\
\text { naissances? }\end{array}$ & $\begin{array}{l}4 \text { ) Est-il acceptable } \\
\text { d'utiliser les méthodes } \\
\text { contraceptives } \\
\text { modernes? }\end{array}$ & $\begin{array}{l}\text { Score de liberté } \\
\text { (Cumul des } \\
\text { réponses } \\
\text { positives) }\end{array}$ \\
\hline Femme & Oui (I)/Non (0) & $\mathrm{I} / 0$ & $\mathrm{I} / 0$ & $\mathrm{I} / 0$ & \\
Mari & $\mathrm{I} / 0$ & $\mathrm{I} / 0$ & $\mathrm{I} / 0$ & $\mathrm{I} / 0$ & $\mathrm{~S}_{\mathrm{f}}=\{0, \ldots, 4\}$ \\
Autres femmes & $\mathrm{I} / 0$ & $\mathrm{I} / 0$ & $\mathrm{I} / 0$ & $\mathrm{I} / 0$ & $\mathrm{~S}_{\mathrm{m}}=\{0, \ldots, 4\}$ \\
Leaders religieux & $\mathrm{I} / 0$ & $\mathrm{I} / 0$ & $\mathrm{I} / 0$ & $\mathrm{I} / 0$ & $\mathrm{~S}_{\mathrm{af}}=\{0, \ldots, 4\}$ \\
$\mathrm{S}$ & & & &
\end{tabular}

La différence entre le score de liberté de la femme $\left(S_{f}\right)$ et son score perçu par rapport à un référent donné $\left(S_{m}, S_{a f}\right.$ ou $\left.S_{\text {Ir }}\right)$ donne sa position relative par rapport à ce groupe de référents. Ces différences permettent de calculer des distances moyennes entre les opinions des femmes et leurs perceptions vis-à-vis de chaque catégorie de référents. Pour une femme i, de score de liberté $S_{i}$ et de score perçu $S_{g}$ (par rapport à un groupe donné g), la valeur de la différence $D_{i}=S f_{i}-S g_{i}$ donne une idée de l'écart entre ses opinions et les attentes perçues du groupe référent $\mathrm{g}$. Elle peut être moins disposée au contrôle des naissances que ce groupe $\left(D_{i}<0\right)$, plus disposée $\left(D_{i}>0\right)$ ou en être en conformité $\left(D_{i}=0\right)$. La distance moyenne $D(f, g)=1 / n \times \sum_{i}^{n}\left|S f_{i}-S g_{i}\right|$ sur l'ensemble de l'échantillon constitue une mesure agrégée de la "distance" moyenne entre la position des femmes et leurs référents du groupe $g$.

\section{Résultats}

\section{Une opinion-type parmi les femmes}

Les femmes s'estiment globalement plus favorables au contrôle de naissances que les trois types de référents sociaux de leur entourage. Elles perçoivent les leaders religieux particulièrement moins ouverts à toute forme d'arbitrage en matière de reproduction. Mais leurs opinions ne sont pas très éloignées de ce qu'elles estiment être celles de leurs maris ou de leurs consœurs de la même communauté. 
Figure 2 - Proportion des femmes favorables ou percevant les référents sociaux favorables aux prérequis d'un contrôle des naissances (en \%)

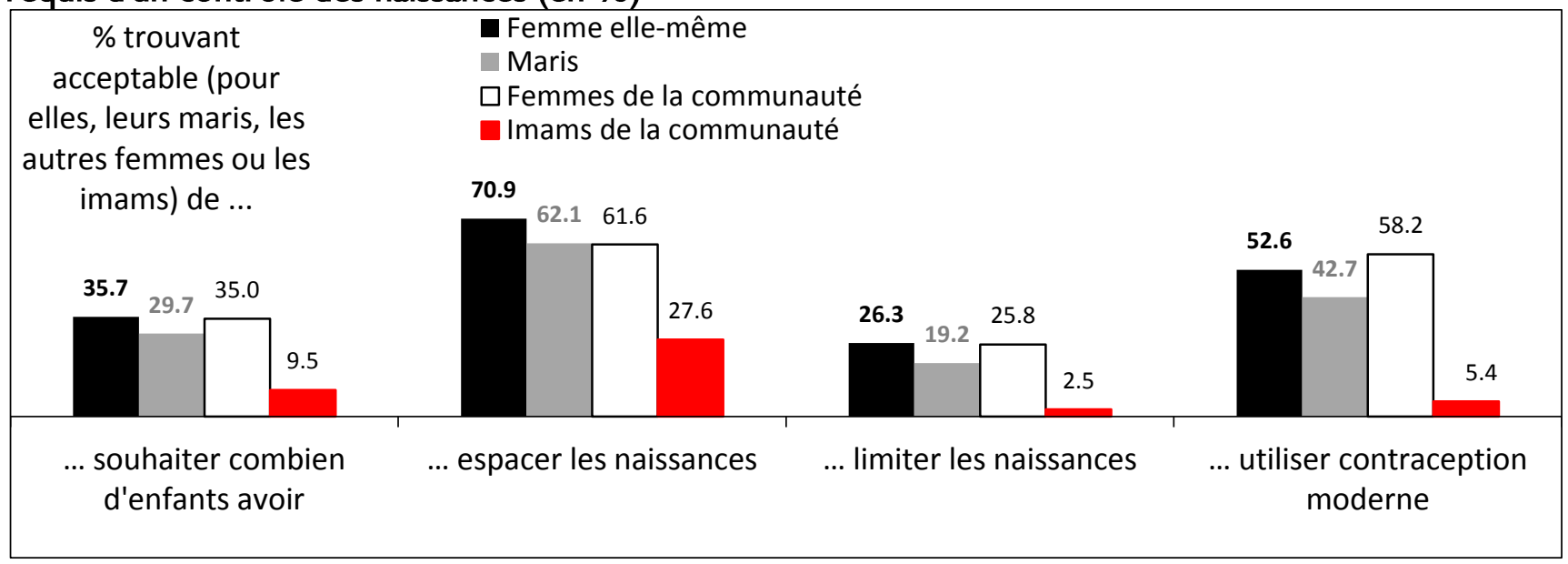

Un ordre se dégage en termes d'acceptation sociale des pré-requis au contrôle des naissances. L'espacement apparait comme la pratique la plus acceptée, suivie par le recours aux méthodes contraceptives modernes. Le choix de la taille de la descendance n'est approuvé que par un tiers des femmes. Une proportion équivalente de femmes estime que leurs maris ou les femmes de leur communauté l'approuvent. La limitation est la forme de contrôle la moins acceptée.

On peut résumer ces constats en deux opinionstype: la première est que l'espacement des naissances en utilisant les méthodes contraceptives modernes est acceptable. La seconde est qu'un projet précis du nombre d'enfants et un recours à la limitation des naissances pour réaliser ce souhait ne l'est pas. L'idée du contrôle des naissances est donc plus ou moins acceptée selon la facette sur laquelle elle est présentée.

Les femmes plus religieuses perçoivent plus souvent les leaders religieux comme favorables au contrôle des naissances
Excepté pour l'espacement, la religiosité ne modifie pas la perception de la femme sur l'opinion des autres femmes. Toujours à l'exception de l'espacement, les femmes moins religieuses sont celles qui mentionnent une position plus favorable de leurs maris. Mais la tendance s'inverse à propos des leaders religieux: les plus religieuses sont en effet celles qui les considèrent plus souvent comme favorables à l'ensemble des quatre traits du contrôle des naissances.

L'espacement des naissances est une dimension particulière suivant laquelle la religiosité a un effet inattendu. Les femmes plus religieuses l'acceptent plus souvent et perçoivent plus souvent l'ensemble des référents sociaux comme favorables à celui-ci. $\mathrm{Ce}$ résultat pourrait s'expliquer en partie par une substitution entre l'acceptation de l'espacement et celle de la limitation; les femmes moins religieuses se montrant en revanche plus favorables et percevant leurs maris plus souvent favorables à la limitation des naissances. 
Figure 3 - Proportion de femmes favorables et trouvant les autres acteurs favorables aux facettes du contrôle de naissances selon la religiosité (en \%)
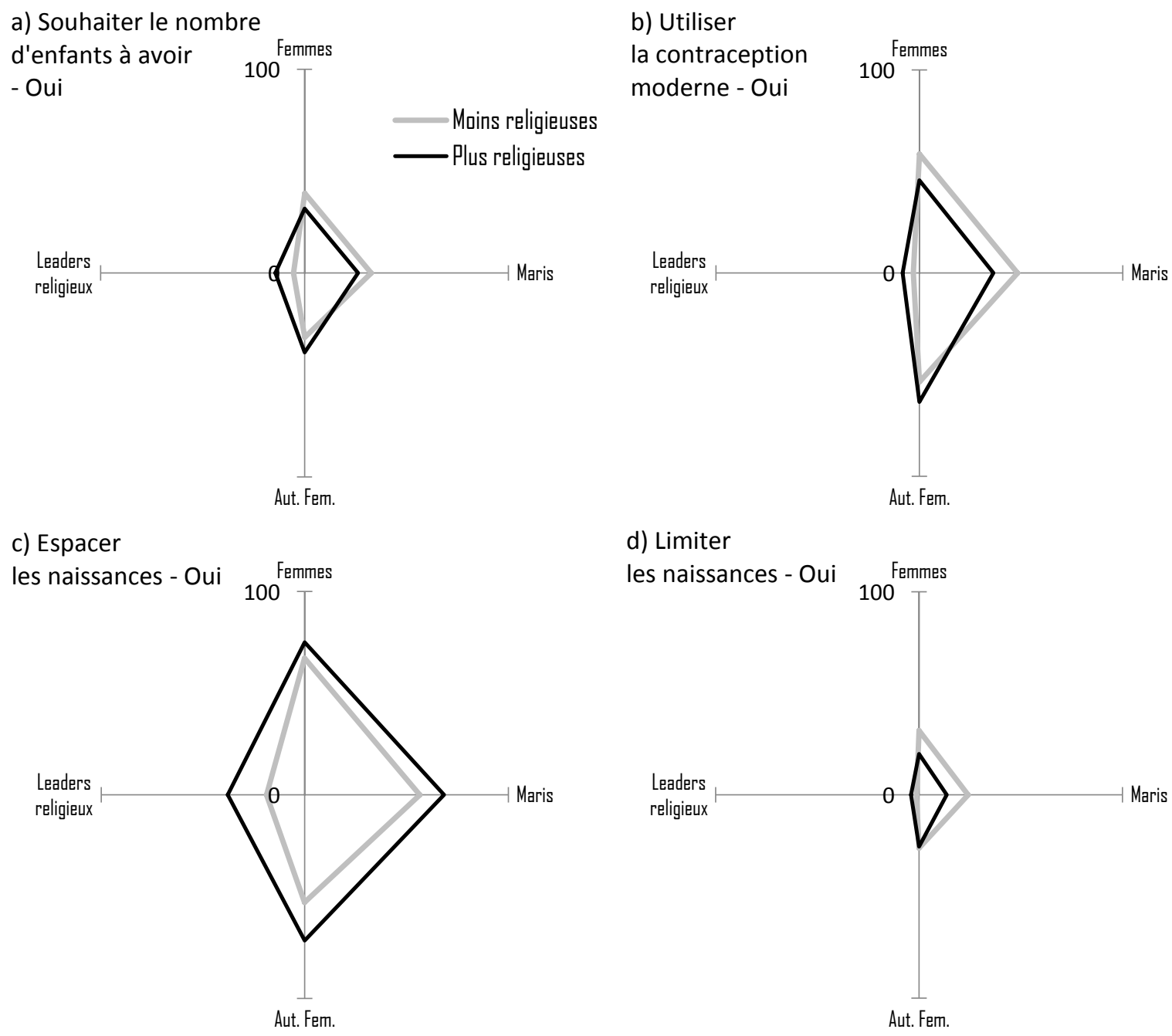

Ces résultats dénotent le rôle complexe du facteur religieux dans la perception des femmes suivant le groupe de référence et les facettes du contrôle de naissances. Au-delà l'opinion de la femme, c'est l'écart entre cette opinion et la perception qu'elle a des opinions des autres qui est porteuse d'information. Le score d'acceptation du contrôle des naissances permet de mesurer cet écart et de synthétiser le rôle de la religiosité.

\section{Religiosité et proximité aux référents sociaux}

D'une manière générale, la distance moyenne entre l'opinion de la femme et sa perception des opinions des référents varie sensiblement avec sa religiosité. L'opinion des femmes plus religieuses est plus proche de leur perception des opinions de leur mari et des leaders religieux. La différence est moins claire par rapport à la perception de l'opinion des autres femmes.

Figure 4 - Distances moyennes entre l'opinion de la femme et sa perception de celles des référents sociaux (sans espacement)

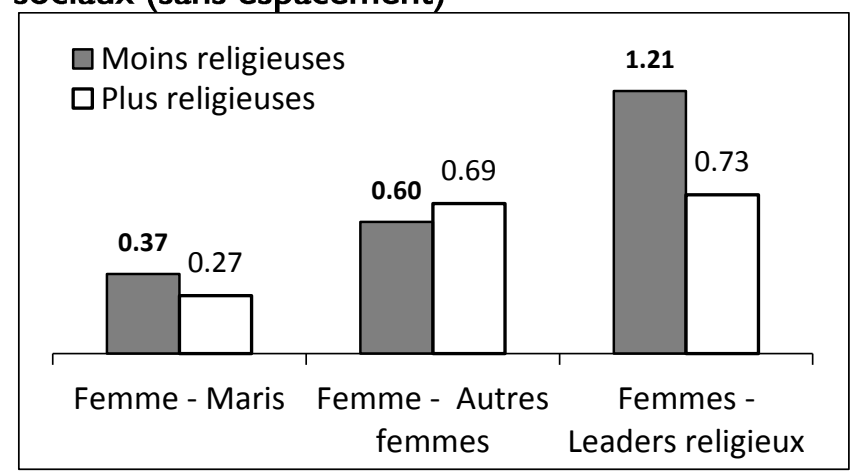

La prise en compte de l'espacement des naissances dans les scores (forme considérée comme la forme la plus acceptable du contrôle des naissances), tend à gommer l'écart selon la religiosité en ce qui est du rapport aux autres femmes de la communauté. Sans l'espacement, les femmes moins religieuses apparaissent plus proches de ces dernières. 
Finalement, pour formuler leurs opinions à propos du contrôle des naissances, les plus religieuses semblent s'orienter plus vers leurs maris et les leaders religieux alors que les femmes moins religieuses s'inspirent plus souvent des femmes de leur entourage. La position relative des femmes par rapport aux référents en termes de score global d'acceptation du contrôle des naissances conforte aussi ces résultats.

Rôle central du mari, formes de contrôle des naissances et religiosité

Les constats sur la place centrale qu'occupe le mari dans les opinions de sa femme à propos du contrôle des naissances se confirment au niveau des analyses multivariées. Bien que la perception que celle-ci a des opinions des autres femmes et des leaders religieux affecte significativement ses opinions, c'est surtout la perception de l'opinion de son mari qui fait la plus grande différence. L'ordre d'importance entre les groupes de référents sociaux établi au niveau descriptif se maintient. La perception d'une opinion favorable des autres femmes améliore les chances pour une femme d'approuver à son tour le contrôle de naissances. Mais c'est la perception de l'opinion du mari qui fait le plus grand effet favorable.

De plus, le niveau d'instruction du mari est positivement associé à une opinion favorable de sa femme, quelle que soit la forme du contrôle des naissances. Par contre, un niveau d'instruction plus élevé de la femme elle-même n'améliore ses chances d'opinions favorables que par rapport aux formes de contrôle les plus "sensibles" (limitation des naissances et utilisation de la contraception moderne). Ces résultats dénotent un dialogue entre la femme et son mari et rappellent que les questions qui touchent à la gestion de la vie reproductive sont d'abord une affaire de couple.

Toutefois, une concordance parfaite entre les opinions de la femme et sa perception des opinions de son mari pourrait aussi s'interpréter comme une certaine aliénation de son libre-arbitre, une sorte de dictature du mari dont les opinions déterminent celles de sa femme.

Tableau 3 - Rapports de chances pour une femme d'avoir une opinion favorable selon sa religiosité, sa perception des opinions des référents et ses caractéristiques socioéconomiques (régressions logistiques)

\begin{tabular}{|c|c|c|c|c|c|}
\hline \multicolumn{2}{|c|}{$\begin{array}{l}\text { [Religiosité }]+ \\
\text { [Perception de l'opinion des référents sociaux] par rapport à } \\
\text { l'espacement (MI), à la limitation (M2), à l'utilisation de la } \\
\text { contraception (M3) et au choix de la taille de la descendance } \\
\text { (M4) + } \\
\text { [Variables socioéconomiques] }\end{array}$} & $\begin{array}{l}\text { MI } \\
\text { Espacement } \\
\text { des } \\
\text { naissances } \\
\text { (Oui) }\end{array}$ & $\begin{array}{l}\text { M2 } \\
\text { Limitation des } \\
\text { naissances } \\
\text { (Oui) }\end{array}$ & $\begin{array}{l}\text { M3 } \\
\text { Utilisation de } \\
\text { la contracep- } \\
\text { tion moderne } \\
\text { (Oui) }\end{array}$ & $\begin{array}{l}\text { M4 } \\
\text { Choix de } \\
\text { la taille de } \\
\text { descendan } \\
\text { ce } \\
\text { (Oui) }\end{array}$ \\
\hline \multicolumn{6}{|c|}{ Religiosité } \\
\hline \multicolumn{2}{|c|}{ Religiosité - Plus religieuses (Moins) } & 0,74 & $0,69 *$ & $0,56 * * *$ & 0,71 \\
\hline \multicolumn{6}{|c|}{ Perception de l'opinion des référents sociaux } \\
\hline \multicolumn{2}{|c|}{ Maris - Oui (Non) } & 26,2 **** & $49,1 * * *$ & 38,7 **** & $112,5 * * *$ \\
\hline \multicolumn{2}{|l|}{ Autres femmes - Oui (Non) } & $6,7 * * *$ & $8,6 * * *$ & $4,3 * * *$ & $4,8 * * *$ \\
\hline \multicolumn{2}{|l|}{ Leaders religieux - Oui (Non) } & $2,3 *$ & 0,8 & $3,7 *$ & $5,2 * *$ \\
\hline \multicolumn{6}{|c|}{ Facteurs socioéconomiques et démographiques } \\
\hline \multicolumn{2}{|c|}{ Milieu de résidence urbain (Rural) } & $\mathrm{I}, 0$ & 0,8 & $\mathrm{I}, 2$ & 0,7 \\
\hline \multicolumn{2}{|c|}{ Age (Continu) } & 1,0 & $\mathrm{I}, \mathrm{I}$ & $\mathrm{I}, 0$ & 1,0 \\
\hline \multicolumn{2}{|c|}{ Nombre enfants nés vivants (Continu) } & $0,9 *$ & $\mathrm{I}, 0$ & $\mathrm{I}, 0$ & $\mathrm{I}, 0$ \\
\hline \multirow{2}{*}{$\begin{array}{l}\text { Niveau d'instruction de la } \\
\text { femme (Aucun) }\end{array}$} & Primaire & 1,2 & 1,2 & 1,3 & 0,9 \\
\hline & Sec. ou supérieur & $\mathrm{I}, 5$ & $1,9 *$ & $2,2 * *$ & 1,4 \\
\hline \multirow{2}{*}{$\begin{array}{l}\text { Niveau d'instruction du mari } \\
\text { (Aucun) }\end{array}$} & Primaire & $1,8 *$ & $\mathrm{I}, 5$ & $\mathrm{I}, 5$ & $\mathrm{I}, 7 *$ \\
\hline & Sec. ou supérieur & $\mathrm{I}, 5$ & $1,9 *$ & $\mathrm{I}, 7 *$ & $\mathrm{I}, 9 *$ \\
\hline \multicolumn{2}{|c|}{ Statut économique élevé (Faible) } & 0,9 & 0,9 & 0,9 & 0,9 \\
\hline \multicolumn{2}{|c|}{ Autonomie - Plus autonomes (Moins) } & $\mathrm{I}, 5$ & 0,8 & 1,3 & 1,2 \\
\hline \multicolumn{2}{|l|}{ Constante } & 0,14 **** & $0,03 * * *$ & $0,09 * * *$ & $0,04 * * * *$ \\
\hline \multicolumn{2}{|l|}{ Observations (n) } & 1739 & 1739 & 1739 & 1739 \\
\hline \multirow{2}{*}{\multicolumn{2}{|c|}{$\begin{array}{l}\text { Pseudo R2 } \\
\text { Chi-deux }\end{array}$}} & 0,68 & 0,64 & 0,66 & 0,77 \\
\hline & & $872 * * *$ & $804 * * *$ & 946 **** & $1146 * * *$ \\
\hline
\end{tabular}

Seuils de significativité: $* * *<0,001 \quad * *<0,0$ I $* 0,05$ 
L'effet de la religiosité sur les opinions de la femme n'est significatif que lorsque ces opinions portent sur deux formes "sensibles" de contrôle de naissances (limitation et contraception moderne), deux formes de contrôle par ailleurs relativement moins approuvées par les femmes plus religieuses. Cela rappelle les raisons de l'opposition islamique au contrôle des naissances soulignées par Roudi-Fahimi (2004 : 5), à savoir les avantages d'une descendance nombreuse pour la Oumma et l'assimilation de toute forme de contrôle des naissances à l'infanticide.

\section{Entourage social, religiosité et culture contraceptive}

Opinions, perceptions, religiosité et expérience contraceptive

D'après notre échantillon d'analyse, $80 \%$ environ de femmes connaissent au moins une méthode contraceptive moderne (89\% à l'EDS 20 I2), sans qu'il n'y ait de différence selon le degré de religiosité. Par contre, en termes d'expérience contraceptive, les femmes moins religieuses ont un légèr avantage. Elles plus souvent déjà utilisé une methode contraceptive moderne (35\%) que les plus religieuses $(27 \%)$.

Cette plus grande expérience parmi les moins religieuses est conforme à leur plus grande acceptabilité de la contraception moderne par rapport aux plus religieuses. Ce résultat peut aussi s'interpréter à la lumière du rôle de la religion dans les liens avec les référents sociaux. En effet, les femmes plus religieuses s'identifient plus à leurs maris et aux leaders religieux, deux types de référents qu'elles perçoivent comme moins favorables à la contraception moderne. La plus grande expérience des moins religieuses est, de ce point de vue, un résultat logique de leur proximité avec leurs consœurs de la même communauté, lesquelles sont plus favorables à la contraception.

Ce léger écart lié à la religiosité disparait lorsqu'on prend en compte l'opinion des femmes sur la contraception. La proportion des femmes ayant déjà utilisé une méthode contraceptive moderne est quatre fois plus importante parmi celles qui approuvent le recours à la contraception que parmi celles qui le désapprouvent. Cet écart est sensiblement le même aussi bien parmi les plus religieuses que les moins religieuses. C'est donc la position de la femme par rapport au recours à contraception - et non sa religiosité - qui déterminerait son expérience contraceptive.

Figure 5 - Proportion de femmes ayant déjà utilisé une méthode contraceptive moderne selon leur opinion à propos de la contraception et la religiosité (\%)

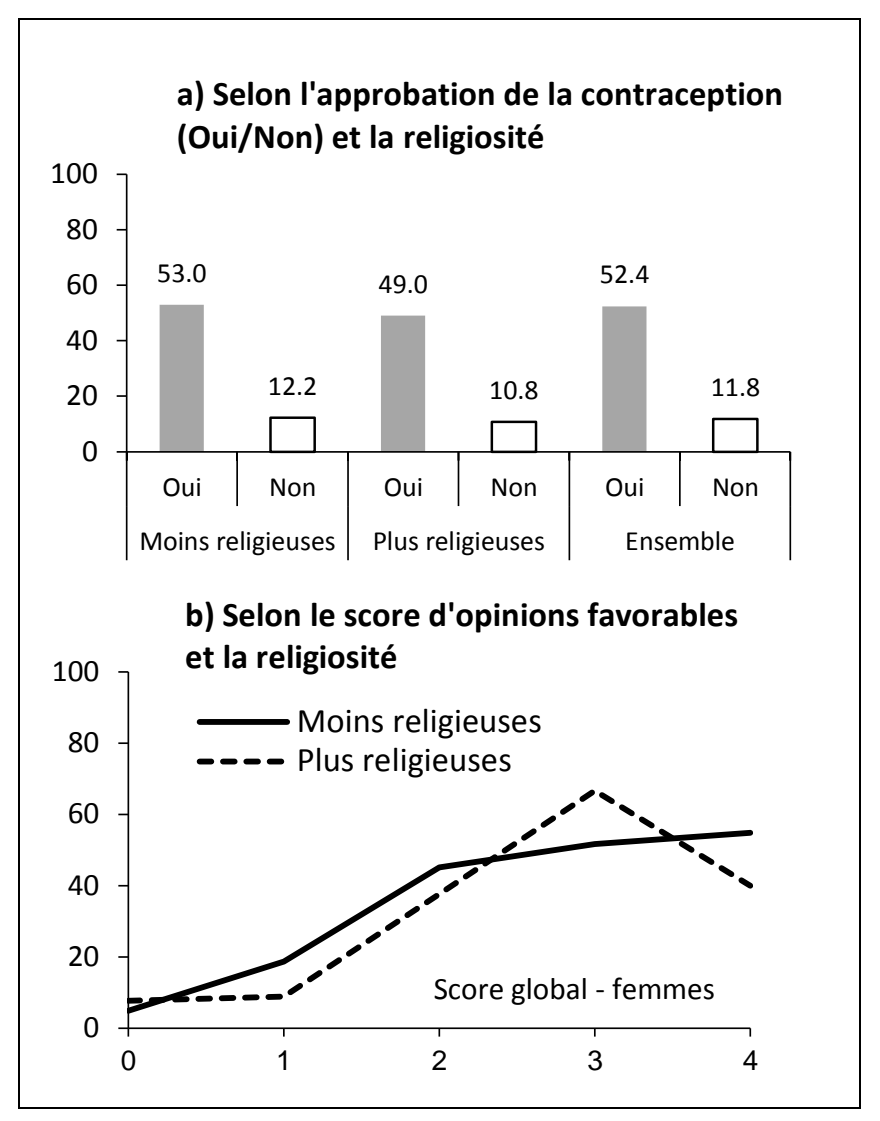

La Figure 5-b le confirme, les opinions des femmes et les attentes perçues de leurs référents sociaux (score global) déterminent mieux leur expérience contraceptive comparativement à la religiosité. D'une manière générale, un score d'opinions favorables plus élevé va de pair avec une plus grande expérience contraceptive. La religiosité n'entraine quasiment aucune différence parmi les femmes peu favorables au contrôle de naissances (score global inférieur ou égal à 2). Par contre, parmi les femmes qui en sont plus favorables (score supérieur à 2), la religion joue un rôle important, sans qu'il ne soit possible de le préciser. En effet, l'avantage des plus religieuses observé parmi celles ayant un score global de 3 fait place à un désavantage parmi celles ayant un score global de 4 . Cela suggère un effet interactif entre le score d'opinions et la religiosité dans l'explication de l'expérience contraceptive.

\section{Rôle central des opinions de la femme}

D'après la théorie du comportement planifié (TCP), les intentions comportementales (ici approchées par les opinions) suffiraient à expliquer le comportement cible (ici utilisation passée et/ou présente de la contraception moderne, c'est-à-dire l'expérience contraceptive). Nous examinons la validité de l'approche TCP en comparant le modèle M7 (dit modèle TCP) au modèle alternatif $M 6$, deux modèles décrits comme suit:

- Le modèle M7 de l'approche TCP met en relation l'expérience contraceptive de la femme avec ses 
opinions sur le contrôle des naissances, telles que prédites $^{\mathrm{x}}$ par les modèles $\mathrm{MI}, \mathrm{M} 2, \mathrm{M} 3$ et M4 estimées dans la première partie de ce travail (tableau 3). Cela suppose que les effets de l'entourage social, de la religiosité et des facteurs socioéconomiques et démographiques transitent par les "intentions" de la femme avant de déterminer son expérience contraceptive.

- Le modèle M6 explique l'expérience contraceptive des femmes par leurs opinions déclarées (proxy des intentions), leur religiosité et leurs caractéristiques socioéconomiques. II suppose que, en plus de leurs effets sur les intentions, les autres facteurs peuvent aussi affecter directement l'expérience contraceptive. C'est l'approche alternative I (AA).

Tableau 4 - Rapports de chances pour une femme d'avoir déjà utilisé une méthode contraceptive selon ses opinions et ses opinions prédites sur le contrôle des naissances (régressions logistiques)

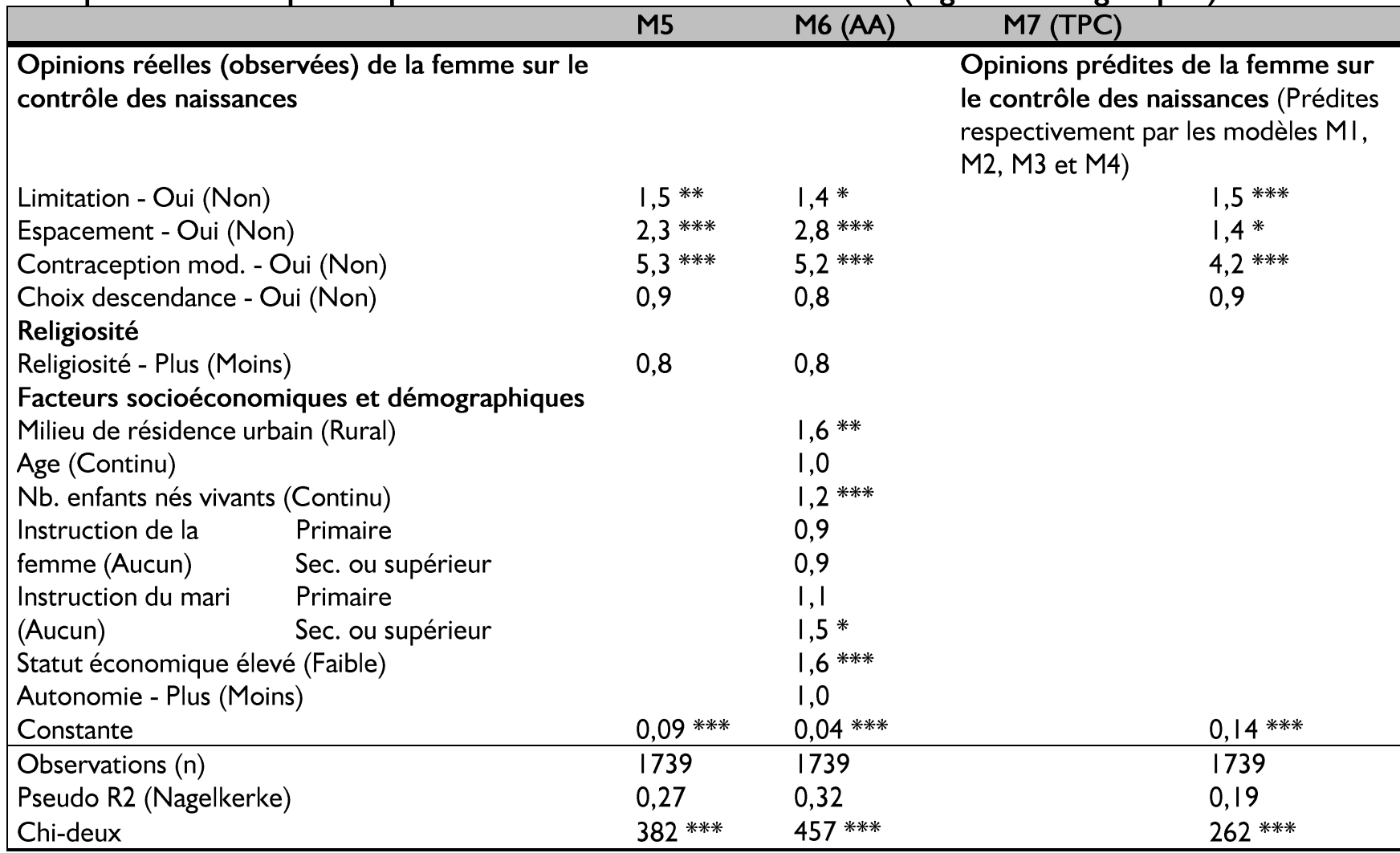

Seuils de significativité: $* * * * 0,001 \quad * *<0,01 \quad *<0,05$

La comparaison des résultats des modèles M6 (AA) et $M 7$ (TCP) suggère que les opinions prédites expliquent globalement bien l'expérience contraceptive des femmes. Les effets des opinions de la femme dans ces deux modèles sont comparables: sauf pour le choix de la taille de la descendance, les femmes qui approuvent une forme de contrôle des naissances font plus souvent recours à la contraception. Cela montre que, connaissant les variables de la norme subjective, la religiosité et les caractéristiques socioéconomiques et démographiques de la femme, on peut déduire ses opinions sur les différentes formes du contrôle de naissances et anticiper par la suite son expérience contraceptive de manière acceptable ( $R 2$ de 0,19 pour $M 7$ contre 0,32 pour $M 6$ ). Ces résultats tendent donc à confirmer l'hypothèse de la TCP, d'autant plus http://aps.journals.ac.za que celle-ci situe la religiosité et les caractéristiques socioéconomiques parmi les facteurs sociétaux de contexte et postule d'emblée que leurs effets sur les comportements transitent par les intentions comportementales, ce que confirme en partie la non significativité de la religiosité au modèle M6.

\section{Discussion}

Nous avons exploré deux principales hypothèses: (I) la primauté de la religion sur l'entourage social en ce qui est de leur influence sur les opinions de la femme à propos du contrôle des naissances et, (2) l'explication de l'expérience contraceptive de la femme par sa religiosité et ses caractéristiques socioéconomiques, au détriment d'une explication par ses seules opinions. 
Contrairement à notre première hypothèse, c'est l'entourage social qui joue le rôle le plus important dans l'opinion de la femme sur le contrôle des naissances, quelle que soit la forme de ce contrôle. La religiosité n'a contribué qu'à l'explication des opinions sur les formes de contrôle que nous avons qualifié de sensibles (limitation des naissances et utilisation de la contraception moderne). Globalement, le rôle de l'entourage social sur les opinions de la femme est de loin plus important que celui de la religiosité, ce qui tend à réfuter notre première hypothèse.

Notre seconde hypothèse n'est pas non plus vérifiée. La religiosité ne joue aucun rôle dans l'expérience contraceptive de la femme et le rôle des facteurs socioéconomiques reste limité. Conformément au schéma explicatif de la TCP, les opinions de la femme, comme proxy des intentions, constituent une explication significative à son expérience contraceptive.

- Méthode contraceptive ou finalité de la contraception?

Le contrôle des naissances renferme un ensemble de pratiques dont certaines sont traditionnellement connues et plus généralement acceptées (abstinence périodique, méthode de l'allaitement maternel et de l'aménorrhée, etc.). D'autres par contre, à l'image des méthodes contraceptives modernes, sont perçues dans certains contextes comme des pratiques importées, étrangères aux habitudes socialement partagées. Dans le contexte nigérien, le débat semble avoir franchi ce cap.

En effet, nos premières analyses montrent que ce n'est pas à la méthode elle-même que les femmes prêtent attention, mais plutôt à la finalité de son utilisation. L'idée d'un contrôle des naissances basé sur le choix d'un nombre précis d'enfants à avoir, s'il le faut, au prix d'une limitation des naissances, n'est pas approuvée par les femmes. Et elles ne la perçoivent pas non plus comme tolérable aux yeux de leur entourage social. II semble que la limitation des naissances renvoie à l'image d'une suspension de la maternité, une image indigeste dans une société où la maternité reste encore très valorisée. A l'opposé, l'idée d'une contraception d'espacement avec un recours aux moyens modernes de contraception est largement acceptée. Les femmes la perçoivent comme acceptable pour leur entourage social également.

\section{- Religiosité et contraception d'espacement}

Cette double signification du concept de contrôle des naissances est encore beaucoup plus palpable en considérant l'influence de la religiosité. Les femmes plus religieuses sont celles qui désapprouvent plus souvent la limitation des naissances et l'utilisation de la contraception moderne. Cela est bien illustré par les protestations des religieux à propos du contenu du document de la CIPD $^{\mathrm{xi}}$ sur la limitation des naissances, des protestations ayant eu des échos jusqu'à Niamey où "le centre de planification familiale a été saccagé et les panneaux publicitaires vantant ses mérites, brûlés" (Locoh et Makdessi, 1996). Par contre, les femmes plus religieuses ne se distinguent pas des moins religieuses sur les questions d'espacement ou de possibilité de choisir la taille de la descendance. Traditionnellement connu et socialement acceptée, l'espacement des naissances semble ne plus être un objet de polémique suivant les clivages religieux et est même généralement accepté parmi les différentes couches socioéconomiques.

\section{- Norme subjective : le rôle de l'entourage}

Les notions de normes subjectives et d'espace de liberté ont permis de montrer la grande influence de l'entourage social sur les opinions de la femme à propos du contrôle des naissances. Dans ses opinions, celle-ci n'est pas insensible aux attentes perçues de son mari, des autres femmes autour d'elle et des leaders religieux. Parmi ces référents, le mari occupe le premier rôle en termes d'influence sur les opinions de la femme à propos du contrôle des naissances. Ce rôle transite par la perception que sa femme a de ses opinions et son niveau d'instruction. Les échanges entre époux peuvent conduire à ce résultat, bien que le dialogue sur la gestion de la fécondité ne soit pas l'apanage des couples sahéliens (Andro et Hertrich, 200I). Dans ce contexte, une forte influence du mari sur les opinions de sa femme pourrait traduire une certaine domination, une référence perpétuelle à la perçeption de ses attentes, une quête de ce qui lui ferait plaisir.

Mais le mari n'occupe pas toujours ce rang de premier référent social, la religiosité apportant des nuances. Si les femmes plus religieuses se réfèrent plus souvent à leur mari et aux leaders religieux, les moins religieuses quant à elles s'inspirent plutôt des autres femmes de leur communauté pour formuler leurs opinions à propos du contrôle des naissances. Les femmes plus religieuses seraient plus respectueuses des enseignements islamiques, lesquels prônent le respect du mari, et peut-être des leaders religieux aussi. Avec une moindre pression perçue des leaders religieux, les femmes moins religieuses sont plus ouvertes envers leurs consœurs de la même communauté. Elles sont plus présentes que les plus religieuses dans certains espaces de socialisation comme les cérémonies, les marchés, la rue, etc., ce qui les rendraient plus enclines à adopter comme modèle de référence les femmes autour d'elles.

\section{- Opinions et culture contraceptive}

Le léger avantage des femmes moins religieuses en matière d'expérience contraceptive souligné au 
niveau descriptif disparait lors des analyses multivariées. Les opinions des femmes s'imposent comme la seule explication clé de leur expérience contraceptive. La religiosité, la norme subjective ainsi que les facteurs socioéconomiques et démographiques n'y contribuent que très peu. $\mathrm{Ce}$ fort lien observé entre l'expérience contraceptive des femmes et leurs opinions à propos du contrôle de naissances peut tout de même souffrir d'une adaptation des opinions au vécu (problèmes d'antériorité); et c'est là une limite de notre postulat des "opinions comme intentions".

\section{Conclusion}

Les femmes tolèrent le contrôle de naissances lorsqu'il s'entend comme un recours à la contraception moderne pour espacer les naissances, mais non comme un choix a priori du nombre d'enfant à réaliser, si nécessaire, au prix de la limitation des naissances. L'entourage social de la femme joue un rôle important sur cette vision du contrôle de naissances. Parmi les référents sociaux qui composent cet entourage, le mari occupe une place de choix, tant par la perception de ses opinions que par son niveau d'instruction. C'est surtout chez les femmes religieuses que les maris, ensemble avec les leaders religieux, restent très influents. Les moins religieuses quant à elles se réfèrent plus souvent aux autres femmes de leur communauté dans leurs opinions. L'expérience contraceptive par contre ne dépend pas de la religiosité mais plutôt des opinions que les femmes se font à propos du contrôle de naissances.

La communication sur la gestion reproductive en destination des femmes peut être améliorée à la lumière de ces résultats. Au sein de certains espaces de socialisation (baptêmes, mariages, marchés, points d'eau, etc.), les consœurs de la même communauté peuvent servir de tremplin pour atteindre et convaincre les femmes à propos de certaines formes du contrôle de naissances, surtout les moins religieuses (environ $75 \%$ de l'échantillon d'analyse) qui fréquenteraient davantage ces espaces.

\section{Références}

Adjamagbo $A$ et Calvès $A-E$ 20I2, "L'émancipation féminine sous contrainte", Autrepart 2, (6I) : 321.

Ajzen, I 199I, "The theory of planned behavior", Organizational Behavior and Human Decision Processes 50, (2): |79-2| I.

Ajzen, I and Klobas, J 2013, "Fertility intentions: An approach based on the theory of planned behavior", Demographic Research 29, (8): $203-$ 232.

Andro, A et Hertrich V 200I, "La demande contraceptive au Sahel: les attentes des hommes se rapprochent-elles de celles de leurs épouses?", Population 5 : 72I-77I.

Bankole, A 1995, "Desired Fertility and Fertility Behaviour among the Yoruba of Nigeria: A Study of Couple Preferences and Subsequent Fertility", Population Studies 49, (2): 317-328.

Campbell, M, Sahin-Hodoglugil N.N, Potts M 2006, "Barriers to Fertility Regulation: A Review of the Literature", Studies in Family Planning 37, (2): 8798.

Casterline, J.B, Zeba A. S. and Minhaj ul H 200I, "Obstacles to contraceptive use in Pakistan: A study in Punjab", Studies in Family Planning 32, (2): 95-II0.

Chamie, J (198I): Religion and Fertility: Arab Christian-Muslim Differentials, Cambridge: University, Sydney.

Chauvin, C, Letirand F and Delhomme P 2007, "Corrélats sociocognitifs de la prise de décision en situation d'interaction dynamique", Le Travail Humain 70, (I): 33-65.

Coale, A.J 1973, "The Demographic Transition Reconsidered," International Population Conference I: 53-72, Liege: International Union for the Scientific Study of Population.

Cotten, N, Stanback J, Maidouka H, Taylor-Thomas J.T and Turk T 1992, "Early Discontinuation of Contraceptive Use In Niger and The Gambia", International Family Planning Perspectives 18, (4): 145-I49.

Goldscheider, C (197I): Population, Modernization, and Social Structure. Boston: Little, Brown.

Institut National de la Statistique (INS) et ICF International 2013, Enquête Démographique et de Santé et à Indicateurs Multiples du Niger 2012, Calverton, Maryland, USA : INS et ICF International.

Johnson-Hanks, J 2005, "When the Future Decides: Uncertainty and Intentional Action in Contemporary Cameroon," Current Anthropology 46, (3): 363-385.

Krauss, S.E, Azimi H.H, Turiman S, Sidek M.N, Rumaya J, Jamiah H.M, Khair ul A.M, Hasnan K and Azma M 2006, "Exploring Regional Differences in Religiosity Among Muslim Youth in Malaysia." Review of Religious Research 3, (47): 238-252.

Mason, K.O 1987, "L'impact du statut social des femmes sur la fécondité dans les pays en développement", in Henri Leridon (eds), Les théories de la fécondité : 447-47I, Paris: Ined. 
Monnier, A 1987, "Projets de fécondité et fécondité effective. Une enquête longitudinale: 1974, 1976, 1979", Population 6: 819-842.

Montgomery, M.R and Casterline J.B 1996, "Social Learning, Social Influence, and New Models of Fertility", Population and Development Review 22: $15 \mid-175$.

Moussa, H 2004, "La pratique de la planification familiale en milieu rural : cas du district de Kollo", Études et Travaux no. 23, Laboratoire d'études et recherches sur les dynamiques sociales et le développement local.

Nagelkerke, N.J.D 1991, "A Note on a General Definition of the Coefficient of Determination, Biometrika 78, (3): 69I-692.

R Core Team 2013, "R: A language and environment for statistical computing", R Foundation for Statistical Computing, Vienna, Austria. URL http://www.R-project.org/.

Régnier-Loilier, A and Vignoli D 20II, "Intentions de fécondité et obstacles à leur réalisation en France et en Italie", Population 66, (2): 40I-43I.

Roudi-Fahimi, F (2004): L'islam et la planification familiale, Policy Brief, Population Reference Bureau.
Rousseaux E and Ritschard G 2013, "The Dataset project: Handling survey data in R", 7th International Conference of Panel Data Users in Switzerland, p. 37-38.

RStudio Team (20|2): RStudio: Integrated Development for R. RStudio, Inc., Boston, MA URL http://www.rstudio.com/.

Sauvain-Dugerdil, C, Douptcheva N, Diawara S.I 2014, "Adoption d'une culture contraceptive et maîtrise du projet familial. La contraception constitue-t-elle une Capabilité?", African Population Studies 28, (2): 854-87I.

Simons, J 1982, "La procréation comme pratique religieuse", in Henri Leridon (eds), Les théories de la fécondité, Paris: Ined : 383-396.

Sounaye, A 20II, "L’Islam au Niger : éviter l'amalgame", Humanitaire 28 - mis en ligne le 02 juillet 20II, consulté le 25 mai 2015. URL: http://humanitaire.revues.org//023.

Stephenson, R and Hennink M 2004, "Barriers to family planning service use among the urban poor in Pakistan", Asia Pacific Population Journal 19, (2): 5-26.

Toulemon, L et Testa M.R 2005, "Fécondité envisagée, fécondité réalisée : un lien complexe", Population et Sociétés 4I5 : I-4.

' L'EDS 2012 estime l'Indice synthétique de fécondité à 7,6 enfants par femme, la prévalence contraceptive chez les femmes de 15-49 ans à 12\% (14\% parmi les femmes en union).

ii "The information set would include a listing and description of other individuals $\mathrm{N}=\{\mathrm{Nj}\}$ whose actions, communications or perceived traits might help person i to resolve uncertainty" (Montgomery and Casterline, 1996).

iii Enquête qualitative que nous avons réalisé au Niger (région de Zinder) en septembre 2015 sur le thème "Projet de famille, qualité de vie et religion".

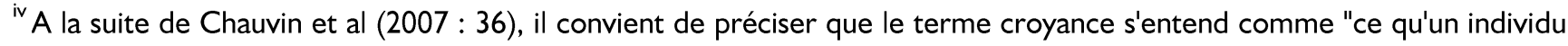
se représente comme étant vrai, étant donné son expérience et ses connaissances".

${ }^{v}$ Hope Consulting est devenu Camber Collective à partir de Juillet 2015. C'est un cabinet de conseil en stratégie au service d'organisations philanthropiques, non-gouvernementales, multilatérales et bilatérales, dans un large éventail de secteurs, dont la santé mondiale, l'intégration financière et l'efficacité du développement (www.cambercollective.com).

vi Pour des raisons d'insécurité, les régions de Tillabéry et de Diffa ont été exclues du plan d'échantillonnage.

vii Glock (196I) propose une définition de "l'objet religieux" basée sur quatre dimensions: une dimension expérientielle traduisant "les sentiments, perceptions et sensations expérimentés par un sujet", une dimension idéologique qui "inclut toutes les représentations sur la nature de la réalité divine", une dimension ritualiste qui "concerne les actes que les gens accomplissent dans le domaine religieux", et une dimension conséquentielle qui touche à "ce que font les gens, ainsi que les attitudes qu'ils adoptent, par suite de leurs croyances, de leurs pratiques et de leurs expériences religieuses". Plus tard, sera ajoutée une cinquième dimension, celle intellectuelle, pour prendre en compte le niveau de connaissance qu'a l'individu des dogmes fondateurs de sa foi (Stark et Glock, 1968).

viii La surreprésentation de la dimension conséquentielle (deux variables) permet de donner plus de poids aux conséquences des connaissances et des pratiques religieuses sur les opinions individuelles. Au-delà du savoir religieux et de sa mise en pratique, l'idée que l'individu se fait de sa marge de liberté en tant que musulman jouerait un rôle important dans ses attitudes et comportements. 
${ }^{i x}$ La validité des scores ainsi définis repose sur deux postulats: l'égalité des poids pour toutes les réponses positives et quelle que soit la dimension de l'approbation, et l'additivité de ces poids pour un groupe donné. Ce second postulat suppose que les quatre questions posées sont porteuses de la même signification à l'égard de l'acceptation du contrôle des naissances. Finalement, qu'ils soient établis à partir des questions initiales ou à partir des scores, les liens entre les opinions de la femme et ses perceptions de la position des référents restent comparables en intensité et en direction, ce qui témoigne de la fiabilité de la mesure du score proposée (Cf. résultats descriptifs).

${ }^{x}$ Les seuils retenus pour déterminer les opinions de la femme à partir des probabilités prédites correspondent au maximum conjoint des critères de sensibilité, de spécificité et de taux de classification (espacement: $p$-seuil = 0,618; limitation: $\mathrm{p}$-seuil $=0$, 150; contraception: $\mathrm{p}$-seuil $=0,360$ et souhait de descendance: $\mathrm{p}$-seuil $=0,200$ ).

${ }^{x i}$ Conférence internationale sur la population et le développement (Caire, 1994). 